\title{
Chile y la pandemia de covid-19: memoria de la medicina social en contexto de crisis neoliberal ${ }^{*}$
}

\author{
Chile and the covid-19 pandemic: remembering social \\ medicine in the context of the neoliberal crisis
}

\author{
Marcelo Sánchez \\ Delgado ${ }^{i}$ \\ ' Profesor asistente, Centro de Estudios \\ Culturales Latinoamericanos/ \\ Universidad de Chile. \\ Santiago de Chile - Chile \\ orcid.org/0000-0002-7697-3699 \\ historia.mjsd@gmail.com
}

Recebido em 14 ago. 2020.

Aprovado em 10 mar. 2021.
SÁNCHEZ DELGADO, Marcelo. Chile y la pandemia de covid-19: memoria de la medicina social en contexto de crisis neoliberal. História, Ciências, Saúde - Manguinhos, Rio de Janeiro, v.29, n.1, jan.-mar. 2022, p.269-276.

\section{Resumen}

Desde el Estallido Social de octubre de 2019 hasta la epidemia de covid-19 en 2020, la sociedad chilena ha vivido un intenso periodo de conflictos sociales y sociosanitarios que han puesto en crisis el modelo de desarrollo neoliberal. La pandemia llegó a evidenciar con fuerza las formas de operar de este modelo en lo que a salud se refiere: prioridad de la ganancia por sobre las vidas, desigualdad, la represión por sobre el diálogo, entre otras. En este contexto de crisis, la memoria de la medicina social, desplegada en Chile como respuesta frente a las epidemias de fines del siglo XIX, resurge como proyecto.

Palabras clave: Chile; covid-19; medicina social; Servicio Nacional de Salud.

\section{Abstract}

Ever since the "social outburst" protests of October 2019 and the covid-19 epidemic of 2020, Chilean society has experienced a period of intense social and sociomedical conflicts that have thrown the neoliberal model of development into crisis. The pandemic powerfully highlighted the ways this model operated in terms of health: it prioritized earnings over lives, inequality, and repression instead of dialogue, among other things. In this context of crisis, remembering social medicine, which was developed in Chile in response to the epidemics of the late nineteenth century, has resurfaced as a project.

Keywords: Chile; covid-19; social medicine; Servicio Nacional de Salud (Chile). 
$\mathrm{E}^{\mathrm{s}}$ n el mes de octubre de 2019 una pequeña alza del precio del metro, como se conoce al transporte subterráneo de Santiago de Chile, levantó algunas protestas aisladas. Algo usual en estos casos, que generalmente daba paso a la resignación. Sin embargo, grupos, al principio minoritarios pero cada vez mayores, de jóvenes de la enseñanza secundaria iniciaron acciones directas evadiendo el pago del boleto. Así, bajo la consigna "Evadir, no pagar, otra forma de luchar", lo que comenzó como intervenciones localizadas terminó desencadenando las llamadas "evasiones masivas" en decenas de estaciones del metro. Su ejemplo de rebeldía fue ridiculizado por las autoridades de gobierno que llamaban a superar el episodio.

En las calles y en las estaciones subterráneas del metro la población comenzó a manifestar su propio descontento, no ya de manera particular frente al alza del costo del transporte, sino frente a problemas profundos, graves y estructurales de la sociedad chilena: fracaso del sistema privado de pensiones, indefensión frente a un sistema de salud segmentado por capacidad de pago, educación de alto costo y educación pública de mala calidad y, tal vez de lo más reiterado por aquellos días, el hecho de vivir en uno de los países menos equitativos del planeta, con desigualdades sociales abismantes (Los 10 países..., 2 jul. 2018). Las manifestaciones tomaron un carácter multitudinario y entre otras frases de combate se escucharon por aquellos días: "porque no son treinta pesos, son treinta años"; es decir, el desborde social que desató esa pequeña alza del costo de transporte se llenó de un sentimiento de injusticia sufrido colectivamente desde la dictadura.

En un desarrollo trágico las manifestaciones alcanzaron cotas de inusitada violencia colectiva y de represión por parte de las fuerzas de orden y seguridad. Pocos días después de las primeras y triviales escaramuzas, se produjeron manifestaciones masivas y el país fue militarizado. En ese escenario decenas de estaciones de la red de metro fueron incendiadas, se dieron saqueos, incendios y vandalismo frente a pequeños y grandes comercios. La represión policial y militar sobre las masas alborotadas provocó en Chile un total de 8.575 víctimas - registradas - de violaciones a derechos humanos (A 4 meses de..., 18 feb. 2020), entre las que se encuentran: violaciones, torturas, asesinatos, desapariciones, traumas oculares - la mayor cantidad de daño ocular por balines registrada a nivel mundial (Rodríguez et al., 2020) -, golpizas, intoxicación y quemaduras por ataques químicos. A esto se le suma la impactante cifra de más de diez mil civiles en prisión y 63 funcionarios policiales imputados por crímenes de lesa humanidad (A 4 meses de..., 18 feb. 2020).

Con el avance del estallido comenzó poco a poco a surgir una demanda ciudadana que haría eco hasta el día de hoy: acabar con la constitución política de la dictadura e iniciar un proceso de Asamblea Constituyente para la redacción de una nueva carta magna. Sin embargo, ante la incontrolable voluntad popular de levantar un proceso constituyente, los partidos tradicionales decidieron crear un pacto entre el oficialismo y los de la llamada "oposición"1 para iniciar un plebiscito constituyente y así apaciguar el frenesí popular. Sin embargo, a pesar del calmante que se intentó inyectar desde el parlamento, la violencia y las manifestaciones no abandonaron las calles de las principales ciudades del país, desde la "marcha del millón" hasta llegar a un paroxismo masivo con la reunión de millones de personas en distintas ciudades por el Día Internacional de la Mujer, el 8 de marzo de 2020. Y entonces apareció en nuestro horizonte el coronavirus. 
El inicio de los contagios fue enfrentado por el ministro de Salud señor Jaime Mañalich, que a poco andar denunciaba como una medida fanática y poco razonable la suspensión de las clases escolares. Esto ocurría prácticamente en el mismo momento en que las municipalidades imploraban a través de los medios de comunicación la necesidad de medidas drásticas de aislamiento. Luego, hubo un enfrentamiento directo entre la autoridad política en materia sanitaria y la directiva del Colegio Médico de Chile, especialmente a través de la voz de su presidenta, la médica Izkia Siches. Los controles sanitarios en los aeropuertos fueron muy débiles y las medidas de algunas autoridades municipales y de mandos medios de la administración resultaban vacilantes y contradictorias. Más temprano que tarde ocurrió lo que ya se sabía cabalmente: alta contagiosidad, diversidad de manifestaciones clínicas y letalidad progresiva ante comorbilidades y vejez. Ante la inminencia de un colapso en los servicios sanitarios se decretó toque de queda nacional, a partir del domingo 22 de marzo, entre las 22 horas horas y las 5 horas. Se instalaron cordones sanitarios alrededor de los centros urbanos más afectados y se dio paso a las llamadas cuarentenas dinámicas, una estrategia fuertemente criticada por la opinión pública y medios independientes, ya que aislaba sectores de la población sin mayor atención a los fenómenos sociales de una gran ciudad latinoamericana como el hacinamiento y la marginalidad de los sectores populares.

A estos factores se sumó el problema de la presencialidad y la estabilidad en el ambiente laboral. Según estudios de PageGroup, un $87 \%$ de las empresas empleaban el teletrabajo, estadística que podría demostrar a primera vista un éxito en la implementación del trabajo a distancia (El 87\% de las..., 30 abr. 2020). Por otra parte, estas cifras sólo nos hablan de aquellos trabajadores y trabajadoras con mayor calificación y desempeño de tareas complejas que residen generalmente en las grandes ciudades. Mirado así, solo 20\% de los(as) trabajadores(as) en Chile pueden realmente acceder a teletrabajo (Teletrabajo..., 13 abr. 2020), mientras que un $80 \%$ de la población trabajadora se veía obligada a movilizarse día a día durante la cuarentena para mantener sus trabajos, situación que facilitó la expansión explosiva de contagios a fines de mayo. Esta realidad se vio agravada con los escándalos de las credenciales de trabajo para el libre desplazamiento, los salvoconductos y el Permiso Único Colectivo (PUC) para traslado de trabajadores de áreas "esenciales" para la economía. Estos documentos otorgaban medios legales a las empresas para poder hacer que sus empleados asistieran presencialmente a sus funciones en zonas de cuarentena sanitaria y, además, les otorgaba la posibilidad de funcionar en horarios de toque de queda. En consecuencia, para junio de 2020, 64.856 empresas habían solicitado permisos para un total de 1.520 .826 empleados, o sea, el 16,6\% de la población en cuarentena poseía un PUC (González, 22 jun. 2020). Además, la situación de los permisos para las empresas no ocurrió sin corrupción e irregularidades, ya que, como relató la periodista Alejandra Matus: "Algunas [empresas] desarrollan actividades no esenciales aprovechando giros incluidos dentro del último instructivo para permisos de desplazamiento, otras obligan a sus trabajadores a ir e incluso hay empresas que piden salvoconductos colectivos invocando giros que no tienen" (González, 22 junio 2020). Por otro lado, la otra solución de las empresas al problema laboral y la incertidumbre económica fueron los despidos masivos, que, amparados por el modelo de flexibilidad laboral, llegaron a la cifra de 2,5 millones de personas en condición de desocupación, un 22\% del total de la fuerza de trabajo de Chile (Ryan, 9 jul. 2020). 
Visto así, las cuarentenas fueron una medida insuficiente y una realidad no exenta de privilegios, ya que el grueso de la masa trabajadora seguía siendo obligada a la presencialidad laboral o bien al despido, mientras que una minoría podía gozar del teletrabajo y la estabilidad laboral.

Con el aumento de contagios y de hospitalizaciones surgió el claro temor a la escasez de respiradores artificiales y a la falta atención sanitaria para la población, ya que con tan sólo poco más de 1.600 respiradores funcionales, de los cuales ya había más de trescientos ocupados, versus los más de 16 mil contagiados que había a fines de abril el colapso era inminente (Realidad..., 24 abr. 2020). En un gesto administrativo inusitado en el modelo neoliberal imperante, a fines de marzo, el Ministerio de Salud tomó un control centralizado de las camas y de los aparatos de respiración mecánica disponibles tanto en los hospitales públicos como en las clínicas privadas. Estas medidas, si bien pueden considerarse legítimamente parte de una política de salud pública, son poco más que una respuesta coyuntural a la pandemia mediante la contabilización y administración temporal de recursos. A pesar de todo, aquellos momentos iniciales de la gestión de la pandemia de covid-19 en Chile fueron tomando ribetes de éxito. Si bien el gobierno llamaba a evitar el triunfalismo y a persistir en las medidas de aislamiento social, todo parecía indicar que Chile lograría "aplanar la curva", mantener a los servicios sanitarios activos, pero no totalmente saturados y gestionar eficazmente el brote.

Ante la primera meseta en la curva de contagios ya se llamaba a una "nueva normalidad" en mayo de 2020. Rápidamente comenzaron los planes para reabrir el comercio y en especial los malls, donde el gobierno esperaba que para "mayo ya debieran estar todos [los malls] bastante habilitados" (Gobierno..., 30 abr. 2020). El plan de la "nueva normalidad" no se llevó a cabo sin duras críticas y oposición. El Colegio Médico de Chile consideró las medidas como imprudentes y que "no es ningún aporte" a la situación actual (Vicepresidente..., 30 abr. 2020), mientras que las alcaldías de diferentes comunas se pusieron "en pie de guerra" contra el gobierno (Baeza, Batarce, 27 abr. 2020). En este contexto, Daniel Jadue, alcalde de Recoleta, interpeló directamente a las autoridades cuando afirmó que "se ha privilegiado la economía por sobre la salud de las personas. El costo se va a pagar en vidas y el gobierno tiene la responsabilidad" (Baeza, Batarce, 27 abr. 2020). Dada las duras críticas y la presión que recibió el gobierno durante este primer período, las autoridades recurrieron a la formación de la Mesa Social Covid-19, un organismo consultivo compuesto por especialistas de la salud, municipios y académicos cuyo fin es (y sigue siendo) impulsar acciones para enfrentar la pandemia. Si bien en la mesa personas críticas al gobierno como Izkia Siches o Ennio Vivaldi, rector de la Universidad de Chile, podían hacer oír su voz, finalmente a la hora de la toma de decisiones, el gobierno tenía diferentes grados de recepción de las propuestas que levantaba la Mesa Social Covid-19, evitando acceder a muchas de sus indicaciones. Visto en perspectiva, la meseta en la curva de casos del mes de mayo fue solo un escalón antes de un ascenso drástico. Más que aplanar la curva, esta se perdió de vista: mayo registró 83.665 casos de los 99.688 contabilizados hasta el momento $(83,92 \%)$ y el $78,46 \%$ de las muertes totales (Retamal, 31 mayo 2020). Con más autoritarismo que diálogo el ministro Jaime Mañalich fue perdiendo credibilidad y apoyo político por su cuestionable gestión durante la pandemia y los(as) casi cinco mil muertos(as) que se apilaban a causa de sus decisiones. 
En consecuencia, el 13 de junio el presidente Sebastián Piñera destituyó a Mañalich de su cargo. Al mes de septiembre de 2020 el exministro era investigado por una comisión del Congreso y las acusaciones en contra de su gestión son graves en términos del manejo y manipulación de la información sobre la pandemia (Cárdenas, 30 sep. 2020).

En su reemplazo, asumió el Ministerio de Salud el señor Enrique Paris, quien impuso desde el primer día de su gestión un giro en la estrategia comunicacional y un refuerzo en las tareas de la salud primaria y en la trazabilidad de los casos. Recién en el mes de agosto los aproximadamente siete millones de habitantes de la capital del país comenzaron a recuperar algunas libertades de tránsito, tras cuarentenas totales impuestas ante el fracaso de las llamadas cuarentenas dinámicas. Al mes de agosto de 2020 Chile contabilizaba más de diez mil personas fallecidas por covid-19, cifra que en noviembre de 2020 se eleva ya sobre las quince mil personas fallecidas, y el país registró uno de los mayores números de contagios por millón de habitantes en el contexto global. A las pérdidas en vidas humanas hay que sumar ahora una crisis de salud mental generalizada, una economía deteriorada y situaciones de extrema precariedad social para la población migrante y los más pobres.

Tras el estallido social de octubre de 2019, la posibilidad y esperanza de cambios al sistema neoliberal imperante fueron puestas en un plebiscito que aprobará o no el paso a la redacción de una nueva constitución. El referéndum estaba planificado para el mes de abril de 2020 y fue reprogramado para el mes de octubre del mismo año. Así, el 25 de octubre las urnas se abrieron para decidir el futuro constitucional del país, donde la opción "Apruebo" ganó con un 78,27\% de los votos. Mientras corría el mes de septiembre de 2020 los casos diarios volvieron a estabilizarse en una meseta de entre mil y dos mil casos detectados al día, dejando atrás por ahora los cinco mil a seis mil casos diarios registrados en junio, y las actividades económicas y sociales comenzaron un proceso de reapertura y reactivación. Finalmente, aunque el sistema integrado de salud pública y privada del país alcanzó en varios momentos casi el total de su capacidad no llegó nunca al temido "triage" de los y las pacientes. Por mi parte, considero que, conviviendo con sus grandes defectos actuales, en las ruinas del sistema público de salud chileno persiste una cultura de servicio y de excelencia en la práctica clínica, que parece revitalizarse ante la urgencia actual. No en vano la medicina social y la responsabilidad del Estado en salud fueron agentes y actores esenciales de los rumbos sanitarios del país en gran parte del siglo XX.

La medicina social chilena logró desplegar y consolidar su tradición local gracias a los médicos que se integraron al Instituto Superior de Higiene creado en 1892, entre otros motivos, para luchar contra las epidemias. En las primeras décadas del siglo XX, el Instituto Superior de Higiene fue un centro de formación informal para los médicos interesados en la higiene pública. Esta primera generación de médicos higienistas impulsó una vigorosa agenda de salud pública que incluye entre sus logros el Código Sanitario de 1918 y la ley de la Caja del Seguro Obrero Obligatorio de 1924.

Sin embargo, a pesar de estos impulsos iniciales, la salud pública chilena de la primera mitad del siglo XX presentaba un panorama trágico que solía subrayarse con una cifra impactante: en la primera mitad del siglo XX, Chile era el país occidental con la más alta tasa de mortalidad infantil (Barnett, 1966). Ese dato, junto a una multitud de evidencias en torno al mal estado sanitario de una gran parte de la población chilena explican en 
parte la urgencia por profesionalizar rápidamente las habilidades de los médicos chilenos en epidemiología y administración pública, rol que cumplió en parte la Escuela de Salud Pública de la Universidad de Chile desde su fundación en 1943.

Además, desde la fundación del Instituto Superior de Higiene en 1892 en adelante, fueron creadas diversas instituciones y legislaciones que iban fortaleciendo y mejorando el rol del Estado chileno en la salud pública. Un paso decisivo en ese camino fue la unificación de servicios e instituciones en una única institución administrada por el Estado, lo que ocurrió con la creación del Servicio Nacional de Salud en 1952. El Servicio Nacional de Salud recogió y profundizó la tarea de prevención y atención sanitaria de la Caja del Seguro Obrero Obligatorio fundada en 1924, de la ley de Medicina Preventiva de 1938, de la Dirección General de Sanidad que ya desde 1918 venía trabajando para la prevención de las epidemias, la sífilis, la tuberculosis y el buen estado sanitario de la nación; es decir, el Servicio Nacional de Salud (SNS) proyectó en forma muy consciente la tradición local de medicina social y durante un poco más de 25 años intentó profundizarla a través de programas que a fines de la década de 1960 incluían la planificación familiar, una ley de salud curativa, salud mental, prevención del alcoholismo, ley de accidentes del trabajo, entre otras áreas en que desplegaba su acción. Según el doctor Hernán Romero - primer director de la Escuela de Salud Pública -, el SNS “representó un paso formidable hacia la socialización de la medicina y hacia una organización única que atienda los problemas de salud de todos los habitantes" (Romero, 1977). Se trataba de un ideal de la medicina social, una aspiración noble y compleja que nunca se alcanzó pero que ponía un norte muy claro: derecho universal a la salud para todos los y las habitantes del país. El SNS tenía también muchos defectos, entre los que se suelen mencionar el desfinanciamiento crónico, la lentitud administrativa, faltas en la coordinación territorial y una atención percibida como "deshumanizada".

Este modelo tuvo un notable desarrollo durante el gobierno de la Unidad Popular (1970-1973) encabezado por el médico Salvador Allende, un socialista con trayectoria en la medicina social, quien sería uno de los mayores promotores de la formación de un sistema de salud de acceso universal y de calidad. Con proyectos tales como el litro de leche para cada niño y niña, construcción de consultorios materno-infantiles, reformas en las jubilaciones, mayor cobertura a la atención médica, mayor inversión en laboratorios médicos, supresión del pago de medicamentos en hospitales, entre otros, el gobierno de la Unidad Popular siguió fortaleciendo el modelo implícito en el Servicio Nacional de Salud, que consistía básicamente en acceso universal y gratuito a los servicios de salud para toda la población.

Todo este sistema comenzó a ser desmantelado a finales de la década de 1970 en el contexto del giro neoliberal de la dictadura militar instaurada en 1973. La dictadura impuso un seguro público, el Fondo Nacional de Salud (FONASA) para quienes quisieran permanecer en la esfera de atención pública y un intermediario financiero en salud privada para los sectores de mayores ingresos, las llamadas ISAPRE (Instituciones de Salud Previsional). Esto generó en la práctica un sistema de salud mixto con distintas calidades para distintas capacidades de pago. En otro golpe radical a la salud pública, el Estado se deshizo de su responsabilidad en atención primaria en salud y la delegó a las municipalidades, con lo que perdió contacto con los problemas cotidianos de salud de la población. 
La posibilidad de comenzar el proceso de una nueva constitución política en 2020 abre en alguna medida el horizonte para retomar lo mejor de la medicina social chilena. Esto debido a que la carta magna actual vela por un estado subsidiario en el área de la salud (y en otras muchas), lo que garantiza el derecho a la libre elección del subsistema de salud al que se quiere optar, ya sea público o privado, pero no se garantiza el derecho a un acceso a salud universal y de calidad. En consecuencia, el sistema actual de salud se ha construido en torno al crecimiento de la atención privada, en cuyo modelo de negocios prima la búsqueda de utilidades para los inversionistas. A la zaga del sector privado está el sistema público, desfinanciado y sobre demandado, con listas de espera para acceder a cirugías y un panorama desolador en la atención de urgencias. Esta concepción neoliberal de la salud contrasta fuertemente las visiones que se tenía antes de la dictadura, en que instituciones - como el Instituto Superior de Higiene de 1892, el Código Sanitario de 1918, la Caja del Seguro Obrero de 1924, la ley de medicina preventiva de 1938, la Escuela de Salud Pública de 1943, el Servicio Nacional de Salud de 1952 y las reformas llevadas a cabo por la Unidad Popular - demostraron con claridad que el rol de lo público en salud es algo fundamental para construir las formas básicas de relación social que hacen posible la vida de la comunidad.

En Chile, como en muchos otros lugares, si bien la pandemia de covid-19 ha instalado dinámicas particulares, ha develado también los problemas sociales de larga duración que ya existían: inequidad, violencia estructural, el abandono del Estado como garante de derechos básicos, alta conflictividad social, problemas críticos en el sistema de pensiones privatizado en dictadura y en las posibilidades de la población para acceder oportunamente a los servicios de salud. Si bien en el contexto latinoamericano se suele destacar la diferencia chilena por su modelo neoliberal con décadas de desarrollo, Latinoamérica como región puede seguir siendo descrita como una unidad en la diversidad. Países latinoamericanos con más presencia del Estado (Argentina, México), con más presencia del mercado (Perú, Chile, Colombia), con desarrollo más o menos centrado en el extractivismo (Brasil, Bolivia), han tenido en estos largos meses de 2020 momentos de fracaso y momentos de complacencia por la tarea bien realizada frente al covid-19. Hasta donde sabemos, Uruguay y Cuba destacan como casos exitosos en el control de la pandemia, mientras que sobre casi todo el resto de los países pesa una enorme duda sobre la legitimidad de las cifras de contagio y muertes. En el trabajo informal como modo de vida para muchas personas y familias, en las migraciones a veces descontroladas y no bien integradas, en la pobreza ineludible a la que han sido arrinconados vastos sectores de la población y en otras tantas realidades de las poblaciones marginales, América Latina presenta notables similitudes.

\section{NOTAS}

* El siguiente texto es una versión más completa, actualizada y sometida a revisión de pares de un post que apareció en el blog de História, Ciências, Saúde - Manguinhos.

${ }^{1}$ En Chile la condición de oposición de las coaliciones y partidos políticos ha sido y sigue siendo cuestionable. Esta situación ha llegado al punto en que la oposición usualmente ubicada en la centro izquierda se ha llegado a denominar la "nueva derecha" o la "otra derecha", ya que su línea política no hace sino defender el modelo neoliberal. 


\section{REFERENCIAS}

A 4 MESES DE inicio del estallido, INDH actualiza cifras de víctimas de violencia y alerta que persisten los casos de lesiones oculares. $E l$ Mostrador, 18 feb. 2020. Disponible en: https:// www.elmostrador.cl/noticias/pais/2020/02/18/a4-meses-de-inicio-del-estallido-indh-actualizacifras-de-victimas-de-violencia-y-alerta-quepersisten-los-casos-de-lesiones-oculares. Acceso en: 30 sep. 2020.

BAEZA, Angélica; BATARCE, Catalina. Alcaldes en pie de guerra con reapertura de los malls. Publimetro, 27 abr. 2020. Disponible en: https:// www.publimetro.cl/cl/noticias/2020/04/27/ alcaldes-pie-guerra-reapertura-los-malls.html. Acceso en: 31 oct. 2020.

BARNETT, Anthony. La especia humana. Ciudad de México: FCE, 1966.

CÁRDENAS, Brisa. Cámara votaría el próximo martes 13 de octubre acusación contra Mañalich: Lo defenderá el abogado Jaime Winter. Radio Sago, 30 sep. 2020. Disponible en: https://www.radiosago.cl/camara-votariael-proximo-martes-13-de-octubre-acusacioncontra-manalich-lo-defendera-el-abogado-jaimewinter. Acceso en: 30 sep. 2020.

EL 87\% DE LAS empresas en Chile están trabajando en modalidad de teletrabajo, según encuesta de PageGroup. AmericaEconomia. com, 30 abr. 2020. Disponible en: https://mba. americaeconomia.com/articulos/notas/el-87de-las-empresas-en-chile-estan-trabajando-enmodalidad-de-teletrabajo-segun. Acceso en: 1 nov. 2020.

GOBIERNO por reapertura de malls: "En mayo ya debieran estar todos bastante habilitados". Tele13, 30 abr. 2020. Disponible en: https:// www.t13.cl/noticia/nacional/gobierno-mallapertura-mayo-coronavirus-30-04-2020. Acceso en: 31 oct. 2020.

GONZÁLEZ, Tomás. Empresas fuera de control: el factor "Permiso Único Colectivo" en la propagación de la pandemia. Diario U Chile, 22 jun. 2020. Disponible en: https://radio.uchile. cl/2020/06/22/empresas-fuera-de-control-elfactor-permiso-unico-colectivo-en-la-propagacionde-la-pandemia. Acceso en: 1 nov. 2020.

LOS 10 PAÍSES más ricos del mundo... y los 10 más desiguales. BBC News Mundo, 2 jul. 2020. Disponible en: https://www.bbc.com/ mundo/noticias-44651569\#: :text=Los\%20 países\%20más\%20desiguales\%20del,son\%20
Sudáfrica $\% 2 \mathrm{C} \% 20 \mathrm{Hait} 1 \% 20 \mathrm{y} \% 20$

Honduras.\&text=imagen $\% 2$ C $\% 20$ Getty $\% 20$

Images-,Pie $\% 20 \mathrm{de} \% 20$ foto $\% 2 \mathrm{C}$,son $\% 20$

Sudáfrica $\% 2 \mathrm{C} \% 20$ Haití $\% 20 \mathrm{y} \% 20$ Honduras. Acceso en: 8 dic. 2020.

REALIDAD de los ventiladores mecánicos en Chile. El siglo, 24 abr. 2020. Disponible en: https://elsiglo.cl/2020/04/24/realidad-de-losventiladores-mecanicos-en-chile. Acceso en: 30 sep. 2020.

RETAMAL, Rodrigo. Mayo, los números del peor mes del coronavirus en Chile: Contabilizó el $83,82 \%$ de todos los casos y el 78,46\% de todos los fallecidos durante la pandemia. $L a$ Tercera, 31 mayo 2020. Disponible en: https:// www.latercera.com/nacional/noticia/mayo-losnumeros-del-peor-mes-del-coronavirus-en-chilecontabilizo-el-8392-de-todos-los-casos-y-el7846-de-todos-los-fallecidos-durante-la-pandemia/OJKLX5ZJORCYTNHNQPONQDHNMU. Acceso en: 30 sep. 2020.

RODRÍGUEZ, Álvaro et al. Ocular trauma by kinetic impact projectiles during civil unrest in Chile. Eye, 24 ago. 2020. Disponible en: https:// doi.org/10.1038/s41433-020-01146-w. Acceso en: 28 sep. 2020.

ROMERO, Hernán. Hitos fundamentales de la medicina social en Chile. In: Jiménez, Jorge (ed.). Medicina social en Chile. Santiago: Ediciones Aconcagua, 1977. p.11-85.

RYAN, Patricia. Lo real es que hay 2 millones 500 mil desempleados en Chile. El Siglo, 9 jul. 2020. Disponible en: https://elsiglo.cl/2020/07/09/ lo-real-es-que-hay-2-millones-500-mildesempleados-en-chile. Acceso en: 31 oct. 2020.

TELETRABAJO en Chile: sólo el 20\% de los trabajadores puede realizarlo y principalmente en Santiago. Agenda País, 13 abr. 2020.

Disponible en: https://www.elmostrador.cl/ agenda-pais/2020/04/13/teletrabajo-en-chilesolo-el-20-de-los-trabajadores-puede-realizarloy-principalmente-en-santiago. Acceso en: 1 nov. 2020.

VICEPRESIDENTE del Colegio Médico: "La apertura de un mall no es ningún aporte". Radio Cooperativa, 30 abr. 2020. Disponible en: https://www.cooperativa.cl/noticias/ sociedad/salud/coronavirus/vicepresidente-delcolegio-medico-la-apertura-de-un-mall-no-esningun/2020-04-30/130518.html. Acceso en: 31 oct. 2020 .

\section{$\rightarrow \rightarrow \rightarrow<<$}

